\title{
Surgical Outcomes and Follow-Up Results of 100 Cases of Laparoscopic Total Gastrectomy Using the Overlap Method with Stapled Closure
}

\author{
Sung Young Park, M.D., In-Seob Lee, M.D., Ph.D., Amy Kim, M.D., Jeong-Hwan Yook, M.D., Ph.D., Byung-Sik Kim, M.D., Ph.D. \\ Department of Surgery, Asan Medical Center, University of Ulsan College of Medicine, Seoul, Korea
}

Purpose: The overlap method is one of the most popular procedures for construction of an esophagojejunostomy and its common entry is usually closed with sutures. This study aimed to report long-term complications and surgical outcomes of the overlap method with stapled closure (OMSC), to compare them with those of laparoscopy-assisted total gastrectomy (LATG), and to analyze a learning curve.

Methods: Between January 2015 and August 2017, 100 consecutive patients underwent laparoscopic total gastrectomy with OMSC for gastric cancer and the patients' medical records were reviewed. Their clinicopathologic characteristics, surgical outcomes, and long-term complications were investigated and compared with those of the LATG group. A learning curve of OMSC was analyzed using the Exponentially Weighted Moving Average chart.

Results: The overall duration of surgery was shorter in the LATG group; however, there was no difference in patients with early gastric cancer. Hospital admission was shorter and the pain scale was lower in the OMSC group. There was no difference in the number of harvested lymph nodes, date of flatus, or incidence of postoperative morbidity. Both groups showed no duodenal stump leakage, anastomosis-related complications, recurrence, or mortality during the follow-up period. Petersen hernia was a notable long-term event following OMSC compared with LATG. At least 27 cases of surgery were required to reach a plateau in terms of surgery duration for OMSC.

Conclusion: OMSC is a safe option for the treatment of gastric cancer and has favorable long-term results and surgical outcomes. Closure of mesenteric defects and Petersen space should be considered.

Keywords: Totally laparoscopic total gastrectomy, Esophagojejunostomy, Overlap method, Stapled closure, Gastric cancer
Received May 27, 2019

Revised 1st July 12, 2019 2nd August 12, 2019

3rd August 22, 2019

Accepted August 26, 2019

Corresponding author

In-Seob Lee

Department of Surgery, Asan

Medical Center, University of Ulsan

College of Medicine, 88 Olympic-ro

43-gil, Songpa-gu, Seoul 05505, Korea

Tel: +82-2-3010-1728

Fax: +82-2-474-9027

E-mail: inseoblee77@gmail.com

ORCID:

https://orcid.org/0000-0003-3099-0140

This is an Open Access article distributed under the terms of the Creative Commons Attribution Non-Commercial License (http:// creativecommons.org/licenses/by-nc/4.0/) which permits unrestricted non-commercial use, distribution, and reproduction in any medium, provided the original work is properly cited.

Copyright ( 2019 The Journal of Minimally Invasive Surgery. All rights reserved.

\section{INTRODUCTION}

Laparoscopic gastrectomy has become an effective surgical option in the treatment of early gastric cancer (EGC) and favorable outcomes, including improved cosmetic appearance, shorter hospital admission, reduced postoperative pain, and improved quality of life, have been reported. ${ }^{1-3}$ With the advancement of instruments and an increase in the laparoscopic skills of surgeons, laparoscopic gastrectomy has evolved from laparoscopy-assisted surgery to totally laparoscopic surgery.

There has been a controversy over the anastomosing method for esophagojejunostomy (EJstomy) in laparoscopic total gas- 
trectomy between using circular and linear staplers. Based on a nationwide survey by the Korean Gastric Cancer Association in 2014, the number of cases of totally laparoscopic total gastrectomy (TLTG) exceeded that of laparoscopy-assisted total gastrectomy (LATG) and anastomosis using a linear stapler, consisting of $70 \%$ of all TLTG cases in Korea. ${ }^{4}$ The overlap method $(\mathrm{OM})$ is one of the most commonly used anastomosis procedures to perform an EJstomy and has the advantage of a reduced mesenteric division, resulting in less tension around the anastomosis compared with the functional end-to-end anastomosing method. ${ }^{5}$ Although some studies have reported the feasibility, safety, and anastomosis-related outcomes of $\mathrm{OM}$, all surgeons closed the common entry of the anastomosis using a suture technique and there is little data for the overlap method with stapled closure (OMSC). ${ }^{5-8}$

We have previously introduced the surgical procedure of the OMSC and reported favorable outcomes. ${ }^{9}$ The present study aimed to report on the long-term complications of our OMSC, as well as the early results of consecutive 100 patients who underwent the procedure; these will be compared with those of LATG, and a learning curve of OMSC will be analyzed.

\section{MATERIALS AND METHODS}

Between January 2015 and August 2017, a total of 107 patients underwent TLTG using the OM for gastric cancer and performed by a single surgeon (-) at the Asan Medical Center (Seoul, Korea). Among them, seven patients who received OM with suture closure were excluded. Therefore, 100 patients were retrospectively investigated in this study. We performed D1 plus lymph node dissection for cT1N0 tumors and D2 or D2-10 lymphadenectomy for the others. For tumors deemed susceptible to an invasion of the subserosa or serosa layer, total omentectomy was also performed.

Following approval by the institutional review board, the medical records of the patients were reviewed to determine demographic and clinicopathologic characteristics, including age at surgery, sex, body mass index (BMI), American Society of Anesthesiologists (ASA) score, tumor size, depth of invasion, number of metastatic, and harvested lymph nodes, TNM stage based on the American Joint Committee on Cancer staging manual 7th edition, surgery duration, postoperative date of flatus, length of hospital admission, numeric rating scale for pain, morbidity, and mortality within 30 days following surgery. In addition, we collected follow-up data following surgery, including the presence of an anastomosis stricture and reflux esophagitis based on the esophagogastroduodenoscopy, history of small bowel obstruction, and recurrence or patient mortality in the postoperative 1 year. To compare surgical outcomes and follow-up results, we also investigated 30 patients with gastric cancer who received LATG using a circular stapler between 2013 and 2014 by the same surgeon. With regard to surgical technique for OMSC, we described procedures containing intracorporeal mesenteric division of jejunum, closure of anastomosis with a linear stapler, and double-stapling reconstruction of jejunojejunostomy in our previous report (Fig. 1A, B). ${ }^{9}$ For
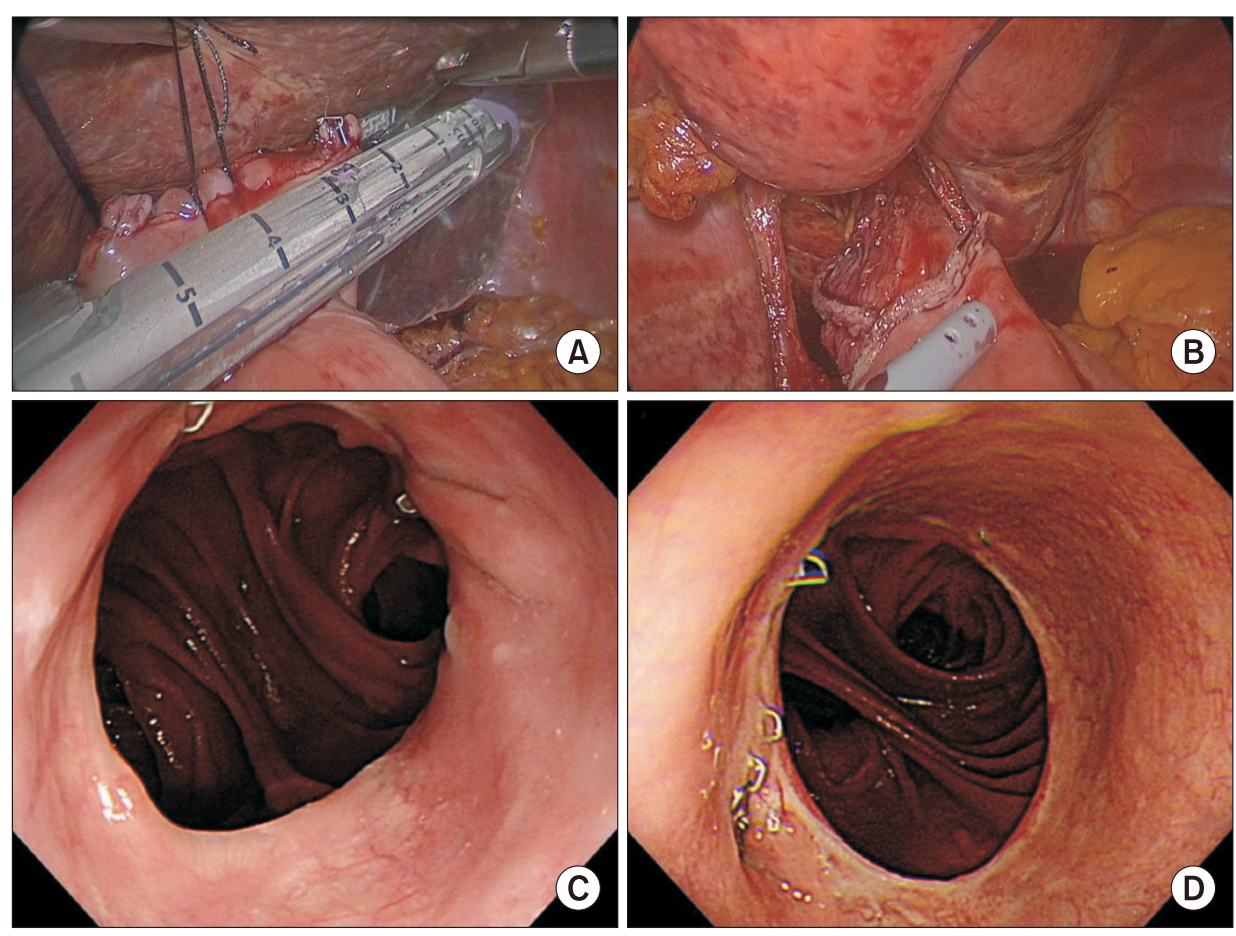

Fig. 1. Closure of esophagojejunostomy with the overlap method with stapled closure $(A, B)$ and postoperative endoscopic finding $(C, D)$. 
LATG, we used five holes incisions, which was the same as TLTG, and added a 7 8-cm sized vertical wound in the epigastrium. The jejunal mesentery was divided and EJstomy was performed similarly to the open total gastrectomy, using a 25$\mathrm{mm}$ sized circular stapler via minilaprotomy, while jejunojejunostomy was performed in a hand-sewing manner.

All patients visited the outpatient clinic for follow-up at 2 3 weeks, and at 3, 6, and 12 months post-surgery; all patients underwent abdominopelvic computed tomography to identify any recurrence or metastasis at 6 and 12 months, and underwent esophagogastroduodenoscopy at 6 or 12 months to evaluate local recurrence or the presence of stricture or reflux esophagitis. Follow-up was conducted until September 2018 and the median follow-up period of OMSC group was 19.4 months (range: 12.1 43.0 months).

\section{Statistical analysis}

The statistical software SPSS version 21.0 for Windows
(IBM Corp., Armonk, NY, USA) was used for all statistical analyses. The comparison of clinicopathologic factors and surgical outcomes between patients were determined using the chi-squared test and independent Student's t-test. One-way analysis of variance was used to compare the mean duration of surgery between patient groups. Statistical significance was set at $p<0.05$. A learning curve was analyzed using the Exponentially Weighted Moving Average chart. ${ }^{10}$

\section{RESULTS}

\section{Comparison of clinicopathologic factors and surgical outcomes between the OMSC and LATG groups}

Patients in the LATG group were younger and had a lower BMI and smaller tumor sizes. More advanced cancer was present in the OMSC group, but this difference was not statistically significant. There was no difference in gender ratio, ASA score, or the number of harvested lymph nodes (Table 1).

Table 1. Comparison of clinicopathologic characteristics of 100 patients with OMSC and 30 cases with LATG

\begin{tabular}{|c|c|c|c|}
\hline Variable & OMSC $(n=100)$ & LATG $(n=30)$ & $p$ value \\
\hline Age (years; mean $\pm S D$ ) & $57.0 \pm 10.3$ & $51.4 \pm 10.3$ & 0.012 \\
\hline \multicolumn{4}{|l|}{ Sex } \\
\hline Male/Female & $56(56.0 \%) / 44(44.0 \%)$ & $13(43.3 \%) / 17(56.7 \%)$ & 0.223 \\
\hline BMI $\left(\mathrm{kg} / \mathrm{m}^{2}\right.$; mean $\left.\pm S D\right)$ & $23.9 \pm 3.3$ & $21.9 \pm 2.4$ & $<0.001$ \\
\hline ASA score & & & 0.609 \\
\hline 1 & $25(25.0 \%)$ & $6(20.0 \%)$ & \\
\hline 2 & $73(73.0 \%)$ & $24(80.0 \%)$ & \\
\hline 3 & $2(2.0 \%)$ & 0 & \\
\hline Tumor size $(\mathrm{cm}$; mean $\pm S D)$ & $3.8 \pm 2.7$ & $2.9 \pm 1.8$ & 0.032 \\
\hline Depth of invasion* & & & 0.074 \\
\hline T1a & $38(38.0 \%)$ & $15(50.0 \%)$ & \\
\hline $\mathrm{T} 1 \mathrm{~b}$ & $35(35.0 \%)$ & $12(40.0 \%)$ & \\
\hline $\mathrm{T} 2$ & $10(10.0 \%)$ & 0 & \\
\hline ТЗ/Т4а & $17(17.0 \%)$ & $3(10.0 \%)$ & \\
\hline No. of harvested lymph nodes (mean \pm SD) & $40.0 \pm 13.0$ & $39.4 \pm 14.9$ & 0.226 \\
\hline TNM stage* & & & 0.282 \\
\hline la & $68(68.0 \%)$ & $27(90.0 \%)$ & \\
\hline $\mathrm{lb}$ & $11(11.0 \%)$ & 0 & \\
\hline$\|$ & $14(14.0 \%)$ & $3(10.0 \%)$ & \\
\hline III & $7(7.0 \%)$ & 0 & \\
\hline
\end{tabular}

OMSC = overlap method with stapled closure; LATG = laparoscopy-assisted total gastrectomy; BMI = body mass index; ASA = American Society of Anesthesiologists score. * Depth of invasion and TNM stage were based on the American Joint Committee on Cancer Staging Manual 7th edition. 
All patients in the LATG group and approximately the initial half of OMSC group started to drink water 24 hours following the operation and a liquid diet was permitted on postoperative day 3. After passing flatus, a soft diet was introduced. The remaining half of the OMSC group began to sip water from the morning of the day following surgery, while a liquid diet and soft diet was permitted on the evening of postoperative days 1 and 2, respectively, according to the introduction of the enhanced recovery after surgery program.

The duration of surgery was shorter in the LATG group (145.3 vs. $158.1 \mathrm{~min}$ ). The duration of hospital admission was shorter (6.6 vs. 7.2 days) and the pain scale was lower in the OMSC group (Table 2). There was no difference in the date of flatus (3.5 vs. 3.6 days). No cases required conversion to open surgery and no anastomosis-related complications, such as leakage, stricture, or duodenal stump leakage, were observed in either group. None of the patients experienced clinically apparent pancreatic fistula or wound problems. Although there was no significant difference in overall incidence of postoperative morbidity, the distribution and severity of complications were different. In the OMSC group, intra-abdominal fluid collection and mechanical ileus were the most common complications, followed by bleeding. All fluid collections were resolved without any additional drain insertion. Three patients experienced small bowel obstruction and, of these, two cases originated from adhesion around the jejunojejunostomy which required bypass surgery. Bleeding from the surgical field and lumen of the jejunojejunostomy occurred in two and one patient, respectively. In the LATG group, one case of fluid collection in the abdominal cavity and one case of mechanical ileus were found, both of which were treated with conservative management. All patients with stage 2 or 3 tumors started adjuvant chemotherapy within 2 months postoperatively. There was no recurrence or disease-related mortality observed during the follow-up period.

\section{Learning curve and differences in duration of surgery according to BMI and depth of invasion in the OMSC group}

We divided the OMSC patients into four groups according to the depth of invasion (EGC vs. advanced gastric cancer,

Table 2. Comparison of surgical outcomes of 100 patients with OMSC and 30 cases with LATG

\begin{tabular}{lccc}
\multicolumn{1}{c}{ Variable } & OMSC $(\mathbf{n}=100)$ & LATG $(\mathbf{n}=30)$ & $p$ value \\
\hline Operation time (min; mean \pm SD) & $158.1 \pm 27.6$ & $145.3 \pm 26.4$ & 0.027 \\
Conversion to open laparotomy & 0 & 0 & 0.423 \\
The postoperative date of flatus (day; mean \pm SD) & $3.5 \pm 0.7$ & $3.6 \pm 0.7$ & 0.047 \\
Hospital stay after operation (day; mean \pm SD) & $6.6 \pm 1.6$ & $7.2 \pm 1.3$ & 0.003 \\
Pain scale & & & 0.066 \\
Postoperative 1st day & $3.7 \pm 0.7$ & $5.0 \pm 2.0$ & 0.003 \\
Postoperative 3rd day & $3.0 \pm 1.5$ & $3.5 \pm 1.4$ & 0.687 \\
Postoperative 5th day & $1.9 \pm 1.2$ & $2.9 \pm 2.1$ & $2(6.7 \%)$ \\
Complication within postoperative 30 days & $9(9.0 \%)$ & 0 & \\
Anastomosis leakage & 0 & 0 & \\
Anastomosis stricture & 0 & 0 & \\
Duodenal stump leakage & 0 & 0 & \\
Luminal bleeding & $1(1.0 \%)$ & 0 & \\
Pancreatic fistula & 0 & 0 & \\
Intra-abdominal bleeding & $2(2.0 \%)$ & $1(3.3 \%)$ & \\
Mechanical ileus & $3(3.0 \%)$ & $1(3.3 \%)$ & \\
Intra-abdominal fluid collection & $3(3.0 \%)$ & 0 & 0 \\
Wound problem & 0 & 0 & \\
Mortality & 0 & 0 & \\
\hline
\end{tabular}

OMSC = overlap method with stapled closure; LATG = laparoscopy-assisted total gastrectomy. 


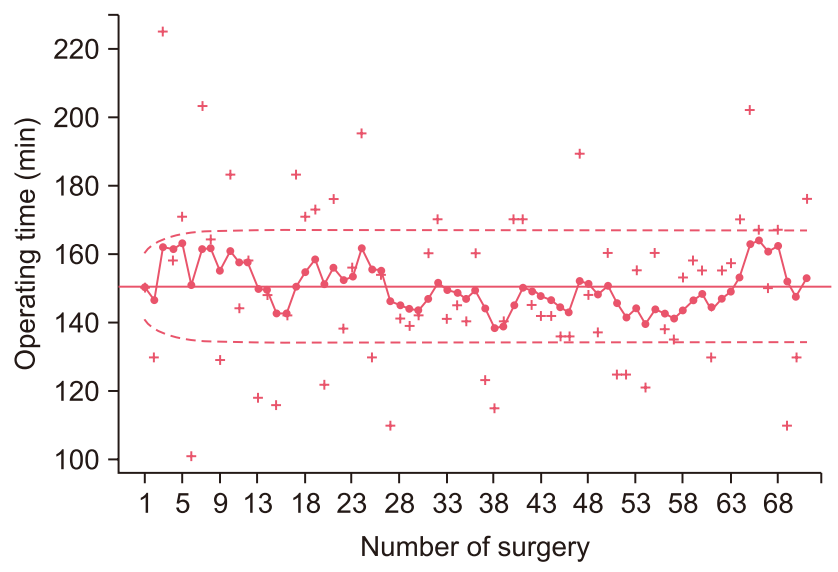

Fig. 2. Learning curve of totally laparoscopic total gastrectomy with the overlap method with stapled closure for early gastric cancer.

AGC) and BMI of 25; 54 EGC \& lower BMI group, 19 EGC \& higher BMI group, 14 AGC \& lower BMI group, 13 AGC \& higher BMI group. The duration of surgery in the four groups was 148.4, 169.8, 160.5, and 178.5 minutes, respectively and there was a significant difference between the groups $(\mathrm{F}=6.784$, $p<0.0001$. To analyze a learning curve in terms of the duration of surgery, we investigated the data of the EGC patients. Twenty-seven cases of surgery were required in total to reach surgical stability in TLTG with OMSC (Fig. 2).

\section{Comparison of long-term complications between the OMSC and LATG groups}

During the follow-up period following surgery, no anastomosis stricture was observed on esophagogastroduodenoscopy, however, 6 7\% reflux esophagitis was seen in both groups (Table 3). The postoperative endoscopic findings of anastomosis following OMSC are shown in Fig. 1C, D. In the OMSC group, $8 \%$ of patients experienced surgery-related problems and, of these, internal hernia was the most common adverse event, followed by mechanical ileus and incisional hernia. Internal hernia occurred through the Petersen space in four patients and the adhesive band related to previous gynecologic surgery in one patient; all of these received surgical correction. Two cases of mechanical ileus improved without surgery. In the LATG group, there were one case of internal hernia and one case of incisional hernia case, for which patients underwent surgery.

\section{DISCUSSION}

Despite evidence of favorable results using a linear stapler in laparoscopic total gastrectomy, there is still no standard anastomosing method owing to technical difficulties in performing
Table 3. Comparison of follow-up results for $>1$ year of patients with OMSC and LATG

\begin{tabular}{|ccc|}
\hline \multicolumn{1}{|c}{ Variable } & OMSC $(\mathbf{n = 1 0 0 )}$ & LATG $(\mathbf{n}=\mathbf{3 0})$ \\
\hline Anastomosis stricture & 0 & 0 \\
Mechanical ileus & 2 & 0 \\
Internal hernia & $5(5.0 \%)$ & $1(3.3 \%)$ \\
Incisional hernia & $1(1.0 \%)$ & $1(3.3 \%)$ \\
Reflux esophagitis & $6(6.0 \%)$ & $2(6.7 \%)$ \\
Los Angeles-grade & & \\
A & 4 & 1 \\
B & 2 & 1 \\
\hline
\end{tabular}

OMSC = overlap method with stapled closure; LATG = laparoscopyassisted total gastrectomy.

an EJstomy. ${ }^{11-13}$ Presently, OM is one of the most favored procedures since it can simplify the establishment of an EJstomy by avoiding the insertion of an intracorporeal or transoral anvil and the placement of purse-string sutures. With regard to totally laparoscopic distal gastrectomy, the stapling closure is popular and its safety and feasibility has been reported in several studies. ${ }^{14-18}$ However, in total gastrectomy, many surgeons prefer a suturing technique to close a common entry after side-to-side anastomosis between esophagus and jejunum, and there is little data on the surgical outcomes of OMSC. To the best of our knowledge, this study is the first one to report on long-term complications and the surgical outcomes of OMSC.

We described certain differences in the procedure in our previous study compared with another reports, one of which is the usage of tagging sutures. These are always used for the esophageal stump, including all layers of esophagus, and they make it easier to manipulate the stump without causing significant injury to the esophagus, while providing clear vision of the esophageal lumen leading to comfortable insertion of a linear stapler. To close a common hole, three or four sutures are placed to prevent slippage of any layer of entry of the anastomosis. To minimize tension around the anastomosis, we divided the jejunal mesentery in the peritoneal cavity. As a result of these procedures, among the 100 patients included in this study, there were no anastomosis-related complications.

Our results demonstrated that the duration of surgery for the OMSC group was longer than that for the LATG group. Regarding extent of lymphadenectomy, surgical treatment for advanced tumors required additional removal of lymph nodes around proper hepatic artery, distal half of splenic artery, and/ or splenic hilum. Moreover, some previous studies reported that operation time was longer in obese patients of BMI over 25 during laparoscopic distal gastrectomy ${ }^{19,20}$ The surgeon 
commenced laparoscopic total gastrectomy as LATG and, in the beginning period, selected patients with EGC and who were not obese. This may explain discrepancies in age and BMI between the two groups and may influence the duration of surgery. The operation time for patients with EGC and a $\mathrm{BMI}<25$ among the OMSC group was $148.8 \mathrm{~min}$, and there was no significant difference compared with that of the LATG group.

Based on the current gastric cancer treatment guidelines, with the exception of node-negative EGC, D2 lymph node dissection is recommended and, in cases of tumors involving the subserosa or serosa layer, total omentectomy is suggested. ${ }^{21}$ Therefore, to analyze a learning curve, we confined patients group into those with EGC requiring D1 plus lymphadenectomy and partial omentectomy and concluded that at least 27 cases of surgery may be required to reach a plateau in terms of the duration of surgery. Previous studies have reported that at least $45 \sim 100$ cases are required to reach a plateau in terms of the duration of surgery and postoperative morbidity. $^{22,23}$ Parameters such as estimated blood loss, incidence of complications, and number of harvested lymph nodes as well as operation time could be used to evaluate improvement of surgeon's skill. We also attempted to analyze a learning curve from the viewpoint of postoperative morbidity or harvested lymph nodes; however, this was unsuccessful due to a lower incidence of complications and steadily similar number of nodes. The initial 30 cases of LATG and more than 150 cases of laparoscopic distal gastrectomy before starting OMSC may be able to affect the learning curve of OMSC and a smaller number of operation cases would be needed compared to another studies.

The present study reported more favorable surgical outcomes, including shorter duration of surgery, length of hospital admission, and a lower morbidity rate, with no serious complications related to the anastomosis or duodenal stump. However, complications related to jejunojejunostomy and $\mathrm{Pe}^{-}$ tersen hernia following TLTG were observed, compared with LATG. Previous studies demonstrated that $5 \sim 8 \%$ of patients experienced internal herniation following Roux-en-Y reconstruction, while the incidence was higher in laparoscopic gastrectomy than open surgery. ${ }^{24,25}$ During the study period, as we only closed the intermesenteric space but not the Petersen space, $4 \%$ of Petersen hernias occurred during the median follow up period of 19.4 months following surgery. Therefore, efforts to repair Petersen or intermesenteric spaces should be considered.

The present study has several limitations, in terms of it being retrospective and data being based on the surgery performed by a single surgeon in a high-volume facility. In addition, although this is a comparative study, it is not a matched one. Despite these limitations, the present study is valuable as it is the first comparative study presenting the favorable longterm complications and surgical outcomes of TLTG using OMSC to treat gastric cancer. TLTG with OMSC is a safe option for the treatment of gastric cancer and has favorable longterm complications as well as surgical outcomes, and at least 27 cases of surgery are required to overcome a learning curve. Care should be taken to prevent internal hernia in terms of long-term events after surgery.

\section{ORCID}

Sung Young Park, https://orcid.org/0000-0001-7780-7759

In-Seob Lee, https://orcid.org/0000-0003-3099-0140

Amy Kim, https://orcid.org/0000-0002-7021-9214

Jeong-Hwan Yook, https://orcid.org/0000-0002-7987-5808

Byung-Sik Kim, https://orcid.org/0000-0001-9579-9211

\section{AUTHORS' CONTRIBUTIONS}

Sung Young Park and In-Seob Lee are responsible for the study concept, design, and writing of the manuscript. Amy Kim and Jeong-Hwan Yook participated in acquisition of data and analysis. Byung-Sik Kim is responsible for critical revision of the manuscript for intellectual content.

\section{CONFLICT OF INTEREST}

None.

\section{FUNDING}

None.

\section{ACKNOWLEDGMENTS}

We thank Dr. Jung-Bok Lee of the Division of Biostatistics and University of Ulsan College of Medicine for statistical consultation and Mr. Chang-Geun Heo, Chun-Myoung Ji, and $\mathrm{Du}-\mathrm{Gi}$ Pin for their assistance with this article.

\section{AVAILABILITY OF DATA AND MATERIALS}

The authors presented all necessary information about the study in the manuscript and do not wish to share the data.

\section{CONSENT FOR PUBLICATION}

Consent to publish was not obtained because an individual person's information is not included in this study. 


\section{ETHICS APPROVAL AND CONSENT TO PARTICIPATE}

The study was approved by the Institutional Review Board of Asan Medical Center. Informed consent was not obtained because this was a retrospective study and there was no person's information included.

\section{REFERENCES}

1) Kim HH, Hyung WJ, Cho GS, et al. Morbidity and mortality of laparoscopic gastrectomy versus open gastrectomy for gastric cancer: an interim report--a phase III multicenter, prospective, randomized Trial (KLASS Trial). Ann Surg 2010;251:417-420.

2) Kitano S, Iso Y, Moriyama M, Sugimachi K. Laparoscopy-assisted Billroth I gastrectomy. Surg Laparosc Endosc 1994;4:146-148.

3) Kim YW, Baik YH, Yun YH, et al. Improved quality of life outcomes after laparoscopy-assisted distal gastrectomy for early gastric cancer: results of a prospective randomized clinical trial. Ann Surg 2008;248:721-727.

4) Information Committee of Korean Gastric Cancer Association. Korean Gastric Cancer Association Nationwide Survey on Gastric Cancer in 2014. J Gastric Cancer 2016;16:131-140.

5) Inaba K, Satoh S, Ishida Y, et al. Overlap method: novel intracorporeal esophagojejunostomy after laparoscopic total gastrectomy. J Am Coll Surg 2010;211:e25-29.

6) Kitagami H, Morimoto M, Nakamura K, et al. Technique of Rouxen-Y reconstruction using overlap method after laparoscopic total gastrectomy for gastric cancer: 100 consecutively successful cases. Surg Endosc 2016;30:4086-4091.

7) Morimoto M, Kitagami H, Hayakawa T, Tanaka M, Matsuo Y, Takeyama H. The overlap method is a safe and feasible for esophagojejunostomy after laparoscopic-assisted total gastrectomy. World J Surg Oncol 2014;12:392.

8) Son SY, Cui LH, Shin HJ, et al. Modified overlap method using knotless barbed sutures (MOBS) for intracorporeal esophagojejunostomy after totally laparoscopic gastrectomy. Surg Endosc 2017; 31:2697-2704.

9) Lee TG, Lee IS, Yook JH, Kim BS. Totally laparoscopic total gastrectomy using the overlap method; early outcomes of 50 consecutive cases. Surg Endosc 2017;31:3186-3190.

10) Vanbrackle LN, Reynolds MR. EVVMA and cusum control charts in the presence of correlation. Commun Stat Simul Comput 1997; 26:979-1008.

11) Bracale U, Marzano E, Nastro P, et al. Side-to-side esophagojejunostomy during totally laparoscopic total gastrectomy for malignant disease: a multicenter study. Surg Endosc 2010;24:2475-2479.

12) Chang KK, Patel MS, Yoon SS. Linear-Stapled Side-to-Side
Esophagojejunostomy with Hand-Sewn Closure of the Common Enterotomy After Prophylactic and Therapeutic Total Gastrectomy. J Gastrointest Surg 2017;21:712-722.

13) Lee IS, Kim TH, Kim KC, Yook JH, Kim BS. Modified techniques and early outcomes of totally laparoscopic total gastrectomy with side-to-side esophagojejunostomy. J Laparoendosc Adv Surg Tech A 2012;22:876-880.

14) Ahn SH, Son SY, Jung DH, Park DJ, Kim HH. Pure single-port laparoscopic distal gastrectomy for early gastric cancer: comparative study with multi-port laparoscopic distal gastrectomy. J Am Coll Surg 2014;219:933-943.

15) Huang $\mathrm{C}$, Lin $\mathrm{M}$, Chen $\mathrm{Q}$, et al. A modified delta-shaped gastroduodenostomy in totally laparoscopic distal gastrectomy for gastric cancer: a safe and feasible technique. PLoS One 2014;9:e102736.

16) Kanaya S, Kawamura $Y$, Kawada H, et al. The delta-shaped anastomosis in laparoscopic distal gastrectomy: analysis of the initial 100 consecutive procedures of intracorporeal gastroduodenostomy Gastric Cancer 2011;14:365-371.

17) Lee HH, Song KY, Lee JS, Park SM, Kim JJ. Delta-shaped anastomosis, a good substitute for conventional Billroth I technique with comparable long-term functional outcome in totally laparoscopic distal gastrectomy. Surg Endosc 2015;29:2545-2552.

18) Motoyama K, Kojima K, Hayashi M, Kato K, Inokuchi M, Sugihara K. beta-Shaped intracorporeal Roux-en-Y reconstruction after totally laparoscopic distal gastrectomy. Gastric Cancer 2014; 17:588-593.

19) Chen K, Pan Y, Zhai ST, et al. Laparoscopic gastrectomy in obese gastric cancer patients: a comparative study with non-obese patients and evaluation of difference in laparoscopic methods. BMC Gastroenterol 2017;17:78.

20) Shimada S, Sawada N, Ishiyama $Y$, et al. Impact of obesity on short- and long-term outcomes of laparoscopy assisted distal gastrectomy for gastric cancer. Surg Endosc 2018;32:358-366.

21) Japanese Gastric Cancer Association. Japanese gastric cancer treatment guidelines 2014 (ver. 4). Gastric Cancer 2017;20:1-19.

22) Jeong O, Ryu SY, Choi WY, Piao Z, Park YK. Risk factors and learning curve associated with postoperative morbidity of laparoscopic total gastrectomy for gastric carcinoma. Ann Surg Oncol 2014;21:2994-3001.

23) Jung DH, Son SY, Park YS, et al. The learning curve associated with laparoscopic total gastrectomy. Gastric Cancer 2016;19:264272.

24) Kelly KJ, Allen PJ, Brennan MF, Gollub MJ, Coit DG, Strong VE. Internal hernia after gastrectomy for cancer with Roux-Y reconstruction. Surgery 2013;154:305-311.

25) Ojima T, Nakamori M, Nakamura M, et al. Internal Hernia After Laparoscopic Total Gastrectomy for Gastric Cancer. Surg Laparosc Endosc Percutan Tech 2017;27:470-473. 